\title{
THE OUTCOME OF CARDIAC OPERATIONS IN INFANTS WEIGHING TWO KILOGRAMS OR LESS
}

Anthony F. Rossi, MD

Howard S. Seiden, MD

Ali M. Sadeghi, MD

Khanh H. Nguyen, MD

Cid S. Quintana, MD

Ronda P. Gross, RN, MSN

Randall B. Griepp, MD
Objective: A review of our recent experience of operating on infants weighing $2 \mathrm{~kg}$ or less who had congenital heart disease was performed to determine the outcome of early surgical repair or palliation. Methods: A retrospective review of hospital records was performed for infants who weighed $2 \mathrm{~kg}$ or less and who were identified to have undergone cardiac operation at our institution January 1992 to June 1997. The data collected included age, weight, gestational age, cardiac diagnosis, surgical procedure, and outcome measures such as length of stay, morbidity, and mortality rate. Outpatient charts were reviewed for follow-up survival and cardiac status. Results: Thirty-three operations were performed on 30 patients. Median age at operation was 19.5 days ( 1 to 140 days), and median weight was $1.8 \mathrm{~kg}$ (1.1 to $2.0 \mathrm{~kg}$ ). Cardiac diagnoses varied, with coarctation of the aorta and tetralogy of Fallot most common. Twenty-four patients were born at 37 or fewer weeks' gestation. Hospital survival was $83 \%$ with no difference in mortality rates based on age, weight, or type of surgical procedure. Premature infants tended to have worse hospital survival. Median postoperative length of stay was 39 days (6 to 122 days). Median duration of mechanical ventilation in survivors was 6 days ( 2 to 24 days). Neurologic complications were documented in eight patients. Of the 25 hospital survivors, $20(80 \%)$ are alive with good cardiac status at a mean follow-up of 13 months. Conclusion: Cardiac operations in a selected group of infants weighing $2 \mathrm{~kg}$ or less can provide acceptable hospital survival. In most instances, complete repair is possible with good medium-term outcome in the survivors. Investigation into neurologic outcomes in these patients is warranted. (J Thorac Cardiovasc Surg 1998;116:28-35)
C ardiac surgery in the neonate with congenital heart disease has progressed dramatically in the past two and a half decades. Inspired by a report of successful correction of congenital heart lesions in infants by Barratt-Boyes and associates ${ }^{1}$ in 1972 , surgeons have made great strides toward making surgical correction of most congenital heart lesions achievable in the neonate. ${ }^{2-9}$ However, low-birthweight infants with congenital heart disease continue to challenge the intellectual and technical skills of those who care for them. The advent of

From the Departments of Pediatrics and Cardiothoracic Surgery, The Mount Sinai Medical Center, New York, N.Y.

Received for publication Oct. 22, 1997; revisions requested Jan. 5, 1998; revisions received Feb. 27, 1998; accepted for publication March 2, 1998.

Address for reprints: Anthony F. Rossi, MD, Division of Pediatric Cardiology, The Mount Sinai Medical Center, Box 1201, 1 Gustave L. Levy Place, New York, NY 10029.

Copyright (c) 1998 by Mosby, Inc.

$0022-5223 / 98 \$ 5.00+0 \quad \mathbf{1 2 / 1 / 9 0 0 1 4}$ alprostadil (prostaglandin $\mathrm{E}_{1}$ ) for the resuscitation of neonates with critical heart disease allows an almost indefinite means of palliation. ${ }^{10}$ Medical palliation with alprostadil for an extended period of time may yield somatic growth in low-birth-weight infants, although this strategy is controversial.

We reviewed our experience in operating on low-birth-weight infants with congenital heart disease. The objective was to determine the outcome of prompt surgical repair or palliation in this subgroup of patients in an attempt to determine the optimal management strategy.

\section{Patients and methods}

Study population. The pediatric cardiovascular database at The Mount Sinai Medical Center was searched for all infants undergoing operations for congenital heart disease from January 1992 to June 1997. Infants whose weight was $2.0 \mathrm{~kg}$ or less at the time of operation were eligible for the study. Infants undergoing ligation of a patent ductus arteriosus as their primary surgical procedure were excluded. Two additional patients were ex- 
Table I. Age, weight, diagnosis, procedure, and hospital survival

\begin{tabular}{|c|c|c|c|c|c|}
\hline Patient & Age (days) & Weight (kg) & Diagnosis & Procedure & Survival \\
\hline 1 & 20 & 1.7 & Coarctation/VSD & $\begin{array}{l}\text { Coarctation repair, } \mathrm{PAB} \Rightarrow \\
\text { complete repair }\end{array}$ & + \\
\hline 2 & 25 & 1.4 & TOF/PA & BTS & + \\
\hline 3 & 4 & 1.3 & TAPVR, obstructed & Repair & + \\
\hline 4 & 19 & 2.0 & TOF & Repair & + \\
\hline 5 & 34 & 1.5 & Coarctation & Repair & + \\
\hline 6 & 1 & 1.4 & TOF & BTS & + \\
\hline 7 & 4 & 1.9 & TAPVR/VSD & Repair & + \\
\hline 8 & 16 & 1.9 & HLHS & Stage I & - \\
\hline 9 & 10 & 1.1 & Coarctation/VSD & Coarctation repair & - \\
\hline 10 & 140 & 1.6 & HLHS & Stage I & + \\
\hline 11 & 13 & 1.8 & Coarctation/VSD & Repair & + \\
\hline 12 & 32 & 1.9 & Coarctation & Repair & + \\
\hline 13 & 30 & 2.0 & Truncus arteriosus & Palliation & + \\
\hline 14 & 43 & 2.0 & Unbalanced AVC & $\mathrm{PAB}$ & + \\
\hline 15 & 8 & 1.9 & HLHS & Stage I & + \\
\hline 16 & 22 & 2.0 & TGA/IVS & $\mathrm{PAB} \Rightarrow$ repair & + \\
\hline 17 & 10 & 1.8 & Coarctation/VSD & Repair & + \\
\hline 18 & 11 & 1.9 & Truncus arteriosus & Repair & + \\
\hline 19 & 8 & 1.8 & TGA/IVS & Repair & - \\
\hline 20 & 27 & 2.0 & TOF & Repair & + \\
\hline 21 & 20 & 1.9 & Heterotaxy & Stage I & + \\
\hline 22 & 16 & 1.9 & Coarctation & Repair & + \\
\hline 23 & 38 & 1.8 & Heterotaxy & BTS & + \\
\hline 24 & 22 & 2.0 & Coarctation & Repair & + \\
\hline 25 & 22 & 1.4 & Unbalanced AVC & $\mathrm{PAB} \Rightarrow \mathrm{DKS} / \mathrm{BTS}$ & - \\
\hline 26 & 5 & 1.6 & PA/IVS & BTS & + \\
\hline 27 & 44 & 1.1 & L-TGA/VSD & $\mathrm{PAB}$ & + \\
\hline 28 & 24 & 2.0 & $\mathrm{TOF} / \mathrm{PA}$ & BTS & - \\
\hline 29 & 14 & 1.5 & TAPVR/VSD & Repair & + \\
\hline 30 & 7 & 1.7 & PA/IVS & BTS & + \\
\hline
\end{tabular}

$A V C$, Atrioventricular canal; $B T S$, Blalock-Taussig shunt; $D K S$, Damus-Kaye-Stansel; $I V S$, intact ventricular septum; $P A$, pulmonary atresia; $P A B$, pulmonary artery band. +, Survival; -, mortality.

cluded because they had had a cardiac operation elsewhere before being seen at our institution. A retrospective review of the hospital and outpatient charts, echocardiogram reports, and cardiac catheterization reports was performed for the remaining 30 patients. Patients' ages and weights at operation and cardiac diagnoses are presented in Table I. Age at operation was 1 to 140 days (median, 19.5 days), and weight was 1.1 to $2.0 \mathrm{~kg}$ (median, $1.8 \mathrm{~kg}$ ). Cardiac diagnoses included coarctation of the aorta (eight patients) and tetralogy of Fallot (TOF; five patients). Eight patients had a variant of single ventricle that would eventually require Fontan palliation. Alprostadil therapy was used before the operation in 22 patients to maintain ductal patency. Nine patients were born at our institution. The remainder were referred to our institution at ages 1 to 95 days (median, 6 days). An operation was performed 1 to 45 days (median, 11 days) after admission to our institution. Three patients underwent two separate cardiac operations while weighing $2.0 \mathrm{~kg}$ or less.

Gestational age at birth was available for 29 patients, with the range for this group 28 to 40 weeks (median, 33 weeks). Twenty-four of the 29 patients were born prematurely (gestational age, 37 weeks or less) and had weights that were appropriate for gestational age. Of the five term infants, one was from a twin gestation; one was born of a mother with severe insulin-dependent diabetes mellitis with vasculopathy; one had multiple congenital anomalies but no defined syndrome, and two had no known explanation for being small for gestational age. Of the entire patient group, four patients were from twin gestations; two patients had heterotaxy syndrome, and one patient each had trisomy 21 , trisomy 18 , a deletion at the $22 \mathrm{q} 11$ locus, nonimmune hydrops fetalis, and the VACTERL association. Five additional patients had a combination of major noncardiac congenital anomalies, including renal, gastrointestinal, skeletal, and abdominal wall abnormalities.

Preoperative strategies. When first seen at our institution and before the operation, the hemodynamic status of each infant was stabilized. This included the use of alprostadil in patients with ductus-dependent heart lesions. Major organ system insults were given time to improve. Nutritional status was optimized with either enteral or parenteral supplementation. An operation was never delayed solely for the purpose of somatic growth.

Surgical procedure. All complete repairs and stage I palliation for hypoplastic left heart syndrome (HLHS) were classified as type A procedures. In newborns who 
were not candidates for eventual biventricular repair, a systemic-pulmonary artery shunt for inadequate pulmonary blood flow and a pulmonary artery band for excessive pulmonary blood flow were also considered type A procedures. Palliative procedures performed in patients who would likely have undergone complete repair had they been larger in size were classified as type B. Thirty-three procedures were performed on 30 patients (Table I). There were 16 open heart operations and 17 closed heart operations performed. Of the 30 initial operations, 22 were type A (definitive procedures) and 8 were type B (palliations). One patient with transposition of the great arteries (TGA) with intact ventricular septum underwent pulmonary artery banding followed by an arterial switch operation. The pulmonary artery banding was classified as a type A procedure because it was not an attempt to palliate the cardiac defect but was part of a rapid, two-stage approach to a definitive repair. ${ }^{12}$ One patient with coarctation and ventricular septal defect (VSD) initially underwent coarctation repair and pulmonary artery banding and could not be weaned from mechanical ventilation. He underwent VSD closure and pulmonary artery debanding 1 month after the initial procedure. One patient with a single ventricle and excessive pulmonary blood flow (unbalanced atrioventricular canal) initially underwent pulmonary artery banding which failed to control symptoms of congestive heart failure. She subsequently underwent a Damus-Kaye-Stansel anastomosis and modified Blalock-Taussig shunt 6 weeks after the initial procedure. Type B procedures included a systemicpulmonary artery shunt for TOF, a pulmonary artery band for an atrioventricular canal defect or VSD, coarctation repair with pulmonary artery banding for coarctation with VSD, and palliation of truncus arteriosus whereby the pulmonary arteries were isolated from the truncus and a systemic-pulmonary artery shunt was placed to provide pulmonary blood flow.

Intraoperative management. All open procedures were done through a sternotomy. Standard aortic and venous cannulas were used, and single venous cannulation was used in all patients. The cardiopulmonary bypass (CPB) circuit was primed with electrolyte solutions (PlasmaLyte; Baxter Healthcare Corp., Deerfield, Ill.) and whole blood to achieve a hematocrit value of $20 \%$ to $25 \%$. Pump flow was calculated to be 150 to $175 \mathrm{ml} / \mathrm{kg} / \mathrm{min}$. Alpha-stat strategy was used. Once CPB was initiated, hypothermia was induced to bring the blood temperature to $10^{\circ} \mathrm{C}$. Before crossclamping, a single dose of potassium chloride, 1.5 to $2.0 \mathrm{mEq} / \mathrm{kg}$, was introduced into the pump circuit to induce diastolic arrest. Hypothermic circulatory arrest was used intermittently or as one single period up to 72 minutes. Before circulatory arrest, the esophageal temperature was brought down to $12^{\circ} \mathrm{C}$ and the head was packed in ice. Hemofiltration and addition of whole blood were carried out on rewarming to produce a hematocrit level above $40 \%$. Recently, modified ultrafiltration was also used. Patients were weaned from CPB with low-dose epinephrine or dopamine, which were generally maintained until extubation.

Postoperative management. Sedation and analgesia consisted of a continuous infusion of fentanyl at a dose of 10 to $15 \mu \mathrm{g} / \mathrm{kg} / \mathrm{hr}$ for all patients for at least the first 24 hours after the operation. Later, sedation and analgesia varied according to the patients' needs. Most infants who required prolonged sedation received fentanyl alone or a combination of fentanyl and midazolam while intubated. Neuromuscular blockade was accomplished with intermittent boluses of vecuronium while the patient was attached to the mechanical ventilator.

Ventilation was accomplished with the Servo Ventilator 900c (Siemens-Elema AB, Solna, Sweden) in all patients initially. Patients received ventilation in the volume control mode with a tidal volume of 15 to $20 \mathrm{ml} / \mathrm{kg}$. The minimum inspired oxygen fraction that provided acceptable arterial oxygen saturations was used. Supplemental oxygen was used when necessary as a pulmonary vasodilator. Ventilator parameters were adjusted to achieve a carbon dioxide tension considered optimal to manipulate the pulmonary vascular resistance.

All patients received perioperative antibiotics. Accounting for the skin flora unique to newborns, the standard antibiotic regimen consisted of a combination of nafcillin and cefotaxime. Patients admitted with an open chest received vancomycin and ceftazidime therapy.

Postoperative management in these infants was otherwise consistent with protocols for managing other neonates after congenital heart operations in our institution. Fluid and electrolyte management included frequent measurements of ionized calcium and potassium. The number of laboratory specimens obtained was similar to that of other infants recovering from heart operations in the intensive care unit. Peritoneal dialysis was not routinely performed but was the preferred modality of dialysis or fluid removal in the presence of renal insufficiency. Infants with HLHS were managed according to a protocol previously described. ${ }^{11}$

Statistics. Student's $t$ test was used to compare length of stay in premature versus term infants and very lowbirth-weight infants versus all others and to compare the gestational age between survivors and nonsurvivors. The Mann-Whitney rank sum test was used to compare age and weight between survivors and nonsurvivors and length of stay between patients who underwent type A versus type B procedures. Fisher's exact test was used to compare survival in premature versus term infants. Confidence intervals (CI) stated are equivalent to 1 standard deviation of the point estimate.

\section{Results}

Mortality. Twenty-five patients survived to discharge (hospital survival rate, $83 \%$; CI, $76 \%$ to $90 \%$ ). There was no statistically significant difference in age, weight, or gestational age in survivors and nonsurvivors of the initial operation (Table II). Three patients who underwent type A procedures died, and two patients who underwent type B procedures died $(p=0.6)$. Three infants died while undergoing open cardiac procedures (mortality rate, $19 \%$; CI, $9 \%$ to $29 \%$ ), and two patients who underwent closed heart operations died (mortality rate, $12 \%$; CI, $4 \%$ to $20 \%$ ). All five patients who died had 
Table II. Comparison of survivors and nonsurvivors

\begin{tabular}{lccc}
\hline & Survivors $(n=25)$ & Nonsurvivors $(n=5)$ & $p$ Value \\
\hline Median age (days) & 20 (range, 1-140) & 16 (range, 8-24) & 0.9 \\
Median weight (kg) & 1.8 (range, 1.1-2.0) & 1.8 (range, 1.1-2.0) & 0.7 \\
Median gestational age (wk) & 33.5 (range, 28-40) & 32 (range, 30-37) & 0.8 \\
Type A procedures (no. of patients) & 19 & 3 & 2 \\
Type B procedures (no. of patients) & 6 & 2 & \\
\hline
\end{tabular}

Table III. Survival (CI) and median and quartiles for postoperative length of stay in survivors

\begin{tabular}{lcccccc}
\hline & & & \multicolumn{3}{c}{ Length of stay (days) } \\
\cline { 4 - 5 } & Survival (\%) & $p$ Value & Median & 25\%ile & $75 \%$ ile & $p$ Value \\
\hline Weight $\leq 1.5 \mathrm{~kg}$ & $75(60-90)$ & 0.6 & 32.5 & 23 & 97 & 0.6 \\
Weight $>1.5 \mathrm{~kg}$ & $86(79-93)$ & & 44 & 12.5 & 72 & 0.9 \\
Gestational age $\leq 37 \mathrm{wk}$ & $79(71-87)$ & 0.6 & 39 & 19.25 & 75.5 & 63 \\
Gestational age $>37 \mathrm{wk}$ & 100 & & 44 & 28.5 & 78.25 & 0.3 \\
Type A procedure & $86(79-93)$ & 0.6 & 44 & 17 & 44 & \\
Type B procedure & $75(60-90)$ & & 31 & 18 & \\
\hline
\end{tabular}

been born prematurely; no deaths occurred in the group of term, small-for-gestational-age infants. Three of the infants who died had shunted single ventricle physiology (mortality rate for patients with this postoperative physiologic condition, $30 \%$; CI, $16 \%$ to $44 \%$ ), accounting for more than one half of all deaths. Among 15 patients with separated pulmonary and systemic circulations, only one death occurred as the result of low cardiac output after an arterial switch operation for TGA (mortality rate, $7 \%$; CI, $1 \%$ to $13 \%)$. The smallest patient $(1.1 \mathrm{~kg})$ had severe pulmonary disease before the operation from a combination of severe congestive heart failure and hyaline membrane disease. This infant had severe hypoxemia and hypercarbia after the operation and died in the operating room.

Length of stay. The length of stay for survivors of the initial operation was 8 to 164 days (median, 57 days). The postoperative length of stay for these patients was 6 to 122 days (median, 39 days). There was no statistically significant difference between the postoperative length of stay in infants who were premature and appropriate for gestational age versus term, small-for-gestational-age infants nor in those who underwent type A versus type B procedures (Table III).

Ventilation. All patients were managed initially as described earlier. Because of significant hypercarbia, two patients were placed on a Sechrist Infant Ventilator model IV-100B (Sechrist Industries, Inc., Anaheim, Calif.) pressure-regulated ventilator. This system may provide less dead space, allowing more effective removal of carbon dioxide. There were no other significant ventilation issues particular to the patients' size. The duration of mechanical ventilation in survivors was 2 to 24 days (median, 6 days). No patient underwent tracheostomy, and all survivors were discharged without supplemental oxygen.

Open chests. Of the 33 surgical procedures, the chest was left open electively at the conclusion of the operation 13 times. All 13 patients with open chests had open heart procedures. Chest closure was performed in the pediatric cardiac intensive care unit 2 to 12 days (median, 5 days) after the initial operation. There were no complications related to having an open chest. Mediastinal cultures were obtained in all patients at the time of chest closure; none were positive.

Infections. Blood cultures were positive in six infants evaluated for septicemia. Isolated organisms included Enterobacter cloacae, Enterococcus faecalis, Staphylococcus aureus, and Pseudomonas aeruginosa. Sterilization of the blood was documented in all cases, and no infant died of sepsis.

Neurologic complications. Intraventricular hemorrhages were documented in five patients; one additional patient had documented hydrocephalus. It was not determined whether these complications were present before the operation. Two additional patients had seizures that were treated with anticonvulsants in the postoperative period.

Other complications. Thrombosis of the superior vena cava was documented in two patients. One of these patients had chronic chylous effusions that persisted until his death 2 months after the operation. A complete atrioventricular block developed 


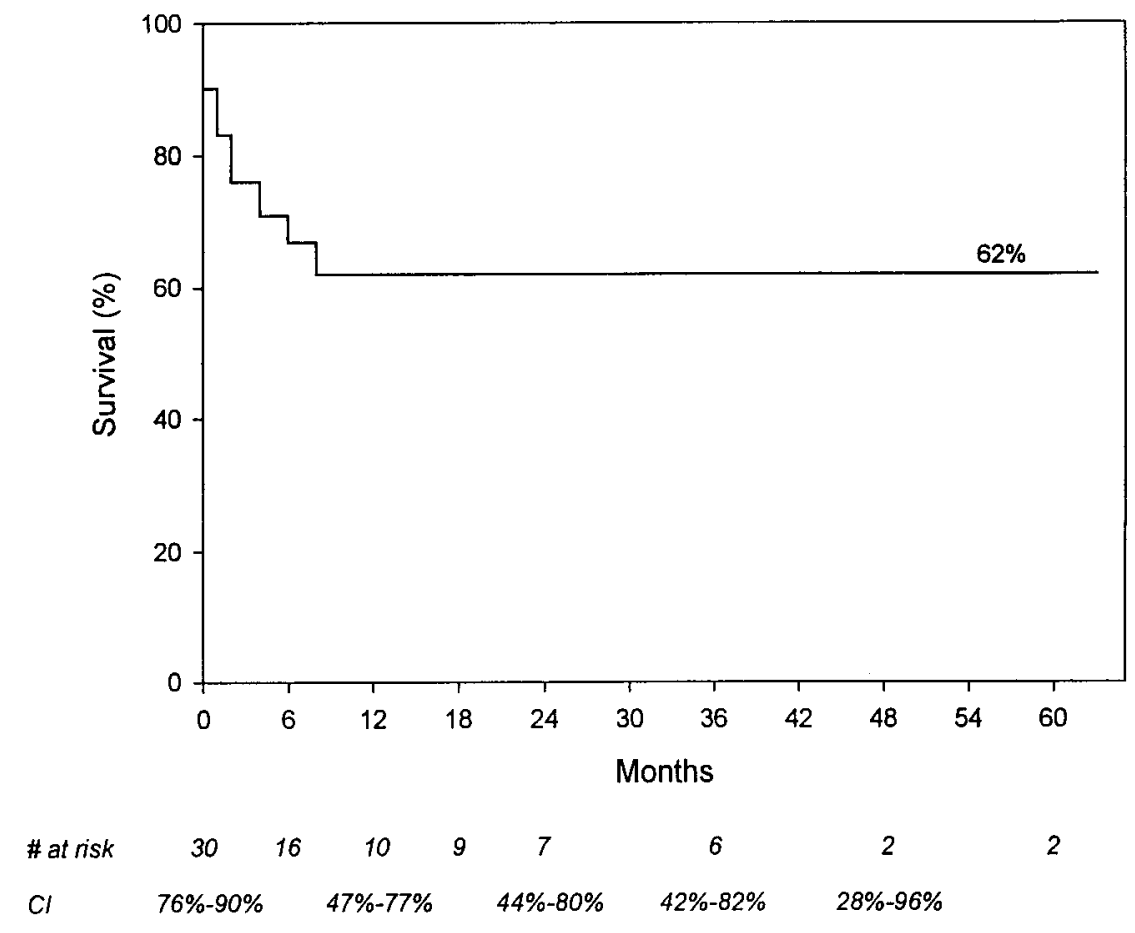

Fig. 1. Kaplan-Meier analysis for survival after heart operations in infants weighing $2 \mathrm{~kg}$ or less. Number of patients at risk and CIs at selected points are also provided.

in one patient after the repair of total anomalous pulmonary venous return (TAPVR) and VSD, which resulted in pacemaker placement before discharge.

Operation in the very low-birth-weight infants. Eight patients weighed $1.5 \mathrm{~kg}$ or less at operation. Three patients had open heart operations, and five patients had closed heart operations. Four procedures were type A, and four procedures were type B. Two deaths occurred in this group of patients. It should be noted that successful reparative open heart operation was achieved in two of these tiny infants, one infant weighing $1.3 \mathrm{~kg}$ with obstructed TAPVR and one infant weighing $1.5 \mathrm{~kg}$ with TAPVR and VSD. However, this group did account for four of the five patients with documented intraventricular hemorrhages and the patient with hydrocephalus. Furthermore, the mean postoperative length of stay in survivors from this group was 54.2 days versus 44.9 days in survivors weighing more than $1.5 \mathrm{~kg}(p=0.6)$.

Follow-up. Of the 25 hospital survivors, 20 patients $(80 \%$; CI, $72 \%$ to $88 \%$ ) are alive with a follow-up of 1 to 63 months (mean, 13 months). Kaplan-Meier analysis is provided in Fig. 1. All survivors were considered to have good to excellent cardiac status at the time of their last outpatient visit, as judged by history, physical examination, and echocardiography.

There were five late deaths of the 25 survivors of the initial operation. One patient with TOF and pulmonary atresia, status post placement of a modified Blalock-Taussig shunt as a newborn, died at 4 months of age on day 1 after operation for complete repair. One child with truncus arteriosus and trisomy 18 died at 17 months of age of an episode of aspiration after having a successful palliation as a newborn and then repair at 10 months of age. One patient with a TOF repair as a newborn died at 4 months of age from complications of biliary atresia. One child appeared well palliated after a bidirectional Glenn shunt for HLHS. A severe respiratory infection developed in this child, who eventually died of that illness. There was no evidence of chronic lung disease in this child before her fatal illness. The final late death occurred suddenly in a 4-month-old infant who had pulmonary atresia with intact ventricular septum and right ventricular-dependent coronary circulation and who underwent a modified Blalock-Taussig shunt in the newborn period.

Of the six surviving infants who underwent type B procedures, four infants have subsequently under- 
gone successful repair of the defect. One patient with coarctation and VSD initially underwent coarctation repair, pulmonary artery banding, and subsequent VSD closure and debanding before discharge because of inability to be weaned from the ventilator. The remaining three patients underwent repair at ages 6 to 23 months for TOF, truncus arteriosus, and an atrioventricular canal defect. One 14-monthold patient died in the postoperative period after repair of TOF with pulmonary atresia. The last patient to undergo a type $B$ procedure is 9 months of age after pulmonary artery banding for L-TGA with VSD and is being observed for spontaneous closure of the VSD.

Nine survivors underwent coarctation repair or aortic arch reconstruction as part of their newborn operation. Recoarctation with a gradient of $35 \mathrm{~mm}$ $\mathrm{Hg}$ developed in one of these patients, who underwent successful balloon dilation. The remaining eight patients have gradients of less than $20 \mathrm{~mm} \mathrm{Hg}$ by blood pressure cuff and/or echocardiographic estimates.

\section{Discussion}

The last 25 years have seen tremendous progress in the surgical management of infants with congenital heart defects. Advancements in technology and forward thinking have led to an evolving philosophy in management strategies. With each advancement, the limitations to palliating or repairing congenital heart defects in small infants diminish. As we push the envelope of surgical management, younger and smaller babies are undergoing heart operations.

The advent of alprostadil has been the most significant therapy developed in the preoperative management of newborns with ductus-dependent congenital heart defects. Noninvasive imaging has progressed to the level where most newborns do not require cardiac catheterization for diagnostic purposes ${ }^{13}$ thereby eliminating associated risks. In fact, no patient underwent cardiac catheterization before the initial operation in this series. The importance of obtaining cardiovascular stability and allowing for the recovery of major organ system insults before operation are now well-accepted philosophies. During the time period of this study, no infant in this weight group was denied operation because of preoperative morbidity. Today's patients are sent to the operating room with better hemodynamic status and with more accurate and detailed diagnoses than ever before.

There has been substantial progress within the operating room as well. Some of the most important developments have been in the area of CPB techniques. ${ }^{14-17}$ Equipment has been streamlined to diminish hemodilution and priming volumes for small infants. Concerns about the newborn's ability to handle the stresses of CPB have been replaced by those concerning fetal bypass techniques. ${ }^{18}$ Surgical modifications have led to improving survivals for reparative congenital heart operations in newborns. Evolving intraoperative strategies include the development of hypothermic circulatory arrest, the development of cold and warm cardioplegia techniques, and a trend toward using low-flow CPB in infants who are undergoing open cardiac operations.

There are significant advantages to a complete repair rather than palliation in the small infant with congenital heart disease. The neonatal heart has been shown to be relatively noncompliant and less capable of handling a volume load than the mature heart. ${ }^{19,20}$ The neonate may therefore be more susceptible to morbidity associated with volume overloading palliations (such as systemic-pulmonary artery shunts) than older infants and children. Furthermore, technical limitations exist in performing systemic-pulmonary artery shunts in small infants. The optimal size of these shunts to achieve ideal acute and chronic systemic-pulmonary blood flow ratios is not known. The possibility of significant pulmonary overcirculation is therefore great with larger shunts. Small shunts have the presumed increased risk of occlusion from thrombosis and the potential for significant distortion of the pulmonary arteries. Elimination of cyanosis in infancy may also have a beneficial effect on development. ${ }^{21}$ Finally, once the palliation is performed, the complete repair will still be necessary at some time. Whether the risk of performing this repair at an older age is diminished is uncertain.

Although it has been our philosophy to perform complete repairs on neonates when possible, eight patients in this series underwent palliations for lesions that were reparable. One patient underwent complete repair before discharge. Two patients had TOF and diminutive pulmonary arteries initially thought to preclude neonatal repair. Two patients weighed $1.1 \mathrm{~kg}$ at operation. One patient had an unbalanced atrioventricular canal defect in whom it was unclear in infancy whether a biventricular repair was possible. The final two patients were very early in the series.

In our experience, managing the subgroup of patients with univentricular physiologic condition 
was particularly difficult. However, small size did not preclude survival. Although low-birth-weight infants with single ventricle physiology may theoretically be at increased risk because of an immature pulmonary vascular bed, the results in this series do not justify waiting to obtain an "ideal" minimal weight before operation. In fact, prolonged exposure to a large left-to-right shunt may increase the risk by increasing the reactivity of the pulmonary vascular bed and exposing the ventricle to a volume overload state before operation. ${ }^{22}$

Eight infants with very low birth weights $(\leq 1.5 \mathrm{~kg})$ comprised $27 \%$ of the patients in the series. Certain issues in the management of this subgroup are worth noting. Most organ systems in this group of patients are immature. Lack of skeletal calcium makes the potential for clinically significant hypocalcemia with low ionized serum calcium problematic. ${ }^{2}$ Temperature regulatory mechanisms are immature, leading to occasional swings in core temperature with its resultant problems. ${ }^{23}$ Renal and hepatic immaturity make fluid management difficult and drug metabolism and excretion erratic. Perhaps most importantly in this population, cardiovascular immaturity, pulmonary underdevelopment, and the immature pulmonary vascular bed make the postoperative management of infants who are in a physiologically transitional stage from fetus to neonate somewhat unpredictable. The immature heart responds differently to loading conditions and is parasympathetically dominant with altered response to $\beta$-adrenergic stimulation. ${ }^{19,20}$ The effects of ventilating immature lung beds in the traditional manner (i.e., high tidal volumes) both acutely and chronically are yet to be evaluated. ${ }^{23}$ The effects of manipulating the developing pulmonary vascular bed with either pharmaceutical or respiratory maneuvers have yet to be described. ${ }^{24-27}$

The hospital survival in this series compares favorably with that reported in a series of 21 infants weighing $2 \mathrm{~kg}$ or less who underwent major cardiac operation from January 1985 to December $1989 .{ }^{28}$ The improved survival may in part be the result of the current series occurring at a later time, taking advantage of some of the advancements discussed earlier. In the earlier series, there was a $100 \%$ mortality rate for patients whose heart defect was part of a recognizable syndrome. The coexistence of a recognizable syndrome was not associated with death in this series. In fact, there were no deaths in the seven patients with a known, defined syndrome. A study of neonates weighing less than $2.5 \mathrm{~kg}$ who underwent open heart operations reported a hospital mortality rate of $16.5 \%,{ }^{29}$ compared with $19 \%$ in our series of open heart patients weighing $2.0 \mathrm{~kg}$ or less. Hospital survival in the current series was similar to that found in infants weighing less than 2.5 $\mathrm{kg}$ who underwent early intervention (i.e., either surgical or transcatheter intervention during their initial hospitalization). ${ }^{30}$ More than one half of that group of 62 patients weighed more than $2.0 \mathrm{~kg}$, and 23 of the 62 patients underwent palliative procedures, making detailed comparisons to the current series difficult.

Limitations. The limitations of this study are primarily related to the retrospective nature of the data collection. The gestational age of one infant was unavailable. The sensitivity of detection of recognizable syndromes and postoperative complications may be suboptimal. There is no discussion of infants who were treated with transcatheter techniques, those who were allowed to grow before the operation, or those who died awaiting operation. The results of this series should be applied only to infants weighing $2.0 \mathrm{~kg}$ or less who have undergone cardiac operation.

\section{Conclusions}

The physiologic limitations to open heart surgery in small and frequently immature infants remains uncertain. However, an aggressive surgical approach in small infants with critical heart disease can be associated with acceptable short- and medium-term survival. The current series demonstrates that cardiac operation in a selected group of infants 1.1 to $2.0 \mathrm{~kg}$ provides hospital survival similar to the early experience in newborns weighing more than $2.0 \mathrm{~kg}$. Furthermore, in most instances, complete repair was an attainable goal in such patients, with acceptable survival and good cardiac status in the survivors with medium-term follow-up. The long-term data regarding neurologic outcome in this group is deficient. Questions regarding the safety of $\mathrm{CPB}$ in preterm, immature infants need to be addressed. One must be particularly concerned with the developing germinal matrix that may be more susceptible to neurologic complications from CPB. Further investigation will consider the neurologic outcome for this unique subgroup of infants.

\section{REFERENCES}

1. Barratt-Boyes BG, Neutze JM, Seelye ER, Simpson M. Complete correction of cardiovascular malformations in the first year of life. Prog Cardiovasc Dis 1972;15:229-53. 
2. Bove EL, Behrendt DM. Open heart surgery in the first week of life. Ann Thorac Surg 1980;29:130-4.

3. Castaneda AR, Mayer JE, Jonas RA, Lock JE, Wessel DL, Hickey PR. The neonate with critical congenital heart disease: repair-a surgical challenge. J Thorac Cardiovasc Surg 1989;98:869-75.

4. Kirklin JK, Blackstone EH, Kirklin JW, McKay R, Pacifico $\mathrm{AD}$, Bargeron LM. Intracardiac surgery in infants under age 3 months: predictors of postoperative in-hospital cardiac death. Am J Cardiol 1981;48:507-12.

5. Kirklin JK, Blackstone EH, Kirklin JW, McKay R, Pacifico $\mathrm{AD}$, Bargeron LM. Intracardiac surgery in infants under age 3 months: incremental risk factors for hospital mortality. Am J Cardiol 1981;48:500-6.

6. Quaegebeur JM, Rohmer J, Ottenkamp J, Buis T, Kirklin JW, Blackstone EH, et al. The arterial switch operation: an eight-year experience. J Thorac Cardiovasc Surg 1986;92: 361-84.

7. Di Donato RM, Jonas RA, Lang P, Rome JJ, Mayer JE, Castaneda AR. Neonatal repair of tetralogy of Fallot with and without pulmonary atresia. J Thorac Cardiovasc Surg 1991;101:126-37.

8. Trowitzsch E, Sluysmans T, Parness IA. Repair of truncus arteriosus during infancy [abstract]. J Am Coll Cardiol 1991;19:110

9. Castaneda AR, Norwood WI, Jonas RA, Colon SD, Sanders SP, Lang P. Transposition of the great arteries and intact ventricular septum: anatomical repair in the neonate. Ann Thorac Surg 1984;38:438-43.

10. Elliott RB, Starling MB, Neutze JM. Medical manipulation of the ductus arteriosus. Lancet 1975;1:140-2.

11. Rossi AF, Sommer RJ, Lotvin A, Gross RP, Steinberg LG, Kipel G, et al. Usefulness of intermittent monitoring of mixed venous oxygen saturation after stage I palliation for hypoplastic left heart syndrome. Am J Cardiol 1994;73:111823 .

12. Jonas RA, Giglia TM, Sanders SP, Wernovsky G, NadalGinard B, Mayer JE, et al. Rapid, two-stage arterial switch for transposition of the great arteries and intact ventricular septum beyond the neonatal period. Circulation 1989; 80(Suppl):I203-8.

13. Huhta JC, Glasgow P, Murphy DJ. Surgery without catheterization for congenital heart defects: management of 100 patients. J Am Coll Cardiol 1987;9:823-9.

14. Subramanian S, Wagner H, Vlad P, Lambert E. Surfaceinduced deep hypothermia in cardiac surgery. J Pediatr Surg 1971;6:612-7.

15. Mori A, Muraoka R, Yokota Y, Okamoto Y, Ando F, Fukumasu H, et al. Deep hypothermia combined with cardiopulmonary bypass for cardiac surgery in neonates and infants. J Thorac Cardiovasc Surg 1972;64:422-9.
16. Pooley RW, Hayer CJ, Edie RN, Gersony WM, Bowman FO, Malm JR. Open-heart experience in infants using normothermia and deep hypothermia. Ann Thorac Surg 1976;22:415-23.

17. Kirklin JK, Westaby S, Blackstone EH, Kirklin JW, Chenoweth DE, Pacifico AD. Complement and the damaging effects of cardiopulmonary bypass. J Thorac Cardiovasc Surg 1983;86:845-57.

18. Reddy VM, Liddicoat JR, Klein JR, Wampler RK, Hanley FL. Long-term outcome after fetal cardiac bypass: fetal survival to full term and organ abnormalities. J Thorac Cardiovasc Surg 1996;111:536-44.

19. Flanagan MF, Fugii AM, Colan SD, Lock JE. Inhibitory effects on myocardial perfusion in pressure overload hypertrophy in immature lambs [abstract]. Pediatr Res 1988;23: 218.

20. Friedman WF. The intrinsic physiologic properties of the developing heart. Prog Cardiovasc Dis 1972;15:87-111.

21. Newburger JW, Silbert AR, Buckley LP, Fyler DC. Cognitive function and age at repair of transposition of the great arteries in children. N Engl J Med 1984;310:1495-9.

22. Iannettoni MD, Bove EL, Mosca RS, Lupinetti FM, Dorostkar PC, Ludomirsky A, et al. Improving results with firststage palliation for hypoplastic left heart syndrome. J Thorac Cardiovasc Surg 1994;107:934-40.

23. Hart SM, McNair M. Gamsu HR, Price JF. Pulmonary interstitial emphysema in very low birthweight infants. Arch Dis Child 1983;58:612-5.

24. Rabinovitch M, Herrere-deLeon V, Castaneda AR, Reid L. Growth and development of the pulmonary vascular bed in patients with tetralogy of Fallot with or without pulmonary atresia. Circulation 1981;64:1234-49.

25. Haworth SG, Hislop AA. Adaptation of the pulmonary circulation to extra-uterine life in the pig and its relevance to the human infant. Cardiovasc Res 1981;15:108-19.

26. Wessel DL, Hickey PR, Hansen DD, Peterson MB. Prostaglandin metabolism and pulmonary vascular response to changes in pCO2 in infants [abstract]. Pediatr Res 1987;21:214.

27. Wessel DL, Hickey PR, Hansen DD. Pulmonary and systemic hemodynamic effects of hyperventilation in infants after repair of congenital heart disease [abstract]. Anesthesiology 1987;67:526.

28. Numa A, Butt W, Mee RB. Outcome of infants with birthweight $2000 \mathrm{~g}$ or less who undergo major cardiac surgery. J Paediatr Child Health 1992;28:318-20.

29. Pawade A, Waterson K, Laussen P, Karl TR, Mee RB Cardiopulmonary bypass in neonates weighting less than 2.5 kg: analysis of the risk factors for early and late mortality. J Card Surg 1993;8:1-8.

30. Chang AC, Hanley FL, Lock JE, Castaneda AR, Wessel DL. Management and outcome of low birth weight neonates with congenital heart disease. J Pediatr 1994;124:461-6. 